\title{
Association of ki67 and tumor marker p53 in locally advanced breast cancer patients and evaluation of response to neoadjuvant chemotherapy: a survey in South Iran
}

This article was published in the following Dove Press journal:

Cancer Management and Research

\author{
Dena Firouzabadi (D) ${ }^{\prime}$ \\ Alireza Rezvani ${ }^{2,3}$ \\ Amirreza Dehghanian (iD) $4,5, *$ \\ Laleh Mahmoudi (iD) ${ }^{1, *}$ \\ 'Department of Clinical Pharmacy, \\ School of Pharmacy, Shiraz University of \\ Medical Sciences, Shiraz, Iran; \\ ${ }^{2}$ Hematology and Oncology Department, \\ School of Medicine, Shiraz University of \\ Medical Sciences, Shiraz, Iran; \\ ${ }^{3}$ Hematology Research Centre, Shiraz \\ University of Medical Sciences, Shiraz, \\ Iran; ${ }^{4}$ Department of Pathology, School \\ of Medicine, Shiraz University of Medical \\ Sciences, Shiraz, Iran; ${ }^{5}$ Trauma Research \\ Center, Shiraz University of Medical \\ Sciences, Shiraz, Iran \\ *These authors contributed equally to \\ this work
}

Purpose: Heterogeneity of breast cancer, the most common cancer in women, complicates approach to its treatment. Neoadjuvant chemotherapy (NAC) in the treatment of locally advanced breast cancer (LABC) with the endpoint of achieving pathologic complete response (pCR) is not always successful. The purpose of this study was to evaluate the clinicopathologic characteristics, biomarker status and response of LABCs to NAC.

Patients and methods: Core biopsies and post-surgical specimens of LABC patients were evaluated after receiving NAC. Their lymph node involvement, tumor staging, grading, size, tumoral and stromal lymphocytic infiltration (TLI, SLI), hormonal status, ki67, p53 and HER2 expression were evaluated. Response to NAC was assessed using pCR, Miller-Payne grading and residual cancer burden.

Results: In a total of 71 patients, pCR rate was 5.6\%. Strong association was observed between ki67 positivity and p53 expression $(P$-value $<0.001)$. Also ki67, TLI and SLI showed association with triple negative tumor subtype ( $P$-value $0.011,0.002$ and 0.014$)$. Good response to NAC was associated with p53 expression. Nodal metastatic residue was also associated with primary tumor's nuclear grade.

Conclusion: Strong correlation of ki67 and p53 can suggest probable interchangeability of both markers in the prognosis of LABC. In this study p53 even showed superiority to ki67 having association with good response. Strong association of ki67, TLI and SLI with triple negative tumor subtype can be parallel to an overall better response rate of this subtype. We can also propose the effectiveness of defining nuclear grade as a prognostic factor towards residual lymph node involvement post NAC.

Keywords: pathologic complete response, tumor proliferation marker, tumoral lymphocytic infiltration, stromal lymphocyte infiltration

\section{Introduction}

Breast cancer is known as the most common cancer in women worldwide. ${ }^{1,2}$ Different approaches including systemic chemotherapy, radiation, surgery and targeted therapy are approved for its treatment. Neoadjuvant chemotherapy (NAC) is the main approach to locally advanced breast cancer (LABC). NAC can help towards shrinkage and down staging of the tumor and its better outcome. ${ }^{3-6}$ Pathologic complete response (pCR) is used to evaluate tumor response to NAC and is known to be associated with patient survival. $^{7-9}$ Based on the American Joint Committee on Cancer (AJCC) staging system, pCR has been defined as the absence of residual invasive tumor in both the 
breast and axillary lymph nodes. ${ }^{4,10}$ Although beneficial in many aspects, NAC is not promising in achieving pCR in lots of patients. Different molecular subtypes have shown different $\mathrm{pCR}$ rates in previous studies. Triple negative tumors and HER2-enriched subtypes have been differentiated from luminal subtypes with regard to their response to NAC and achieving $\mathrm{pCR}^{2,11-13} \mathrm{~A}$ nuclear protein known as a proliferation marker that is detected during cell cycle except for the G0 resting phase is ki67. Its high levels can indicate greater tumor progression and on the contrary better response to chemotherapy. ${ }^{12,14-16}$ However the unavailability of a consensus on the cut-off point for ki67 evaluation, has restricted its use as a predicting factor and therefore defining its eligibility for prediction of response to NAC seeks further studies. ${ }^{17,18}$ A tumor suppressor gene-related protein that is involved in natural apoptosis of cells is p53. Its expression can be used as another predictor of response to therapy; however conflicting results have been seen in this regard in past studies. ${ }^{15,19-21}$ Heterogeneity of breast cancer urges researchers to discover more about its tumor characteristics and factors affecting response to chemotherapy. Since no relevant data has been available to date from LABC patients in Iran, in this retrospective study we decided to evaluate the molecular status, histologic properties, and response to NAC in LABC patients, at our institution.

\section{Materials and methods}

\section{Patient selection}

After inspecting the medical records of 412 breast cancer patients from April 2014 to June 2018, data of 172 stage III breast cancer patients were extracted from archived data of the pathology department of Shahid Faghihi hospital, a large referral hospital in Shiraz, South Iran, affiliated to Shiraz University of Medical Sciences. After further evaluations, 71 LABC patients according to the National Comprehensive Cancer Network (NCCN) guidelines, ${ }^{22}$ with available core biopsies and resected pathology specimens, were selected. The inclusion criterion of receiving standard neoadjuvant chemotherapy before surgery was considered. Patients with confirmed metastasis at the time of diagnosis and also inflammatory carcinoma were excluded. Patients were selected regardless of the chemotherapy agents used and number of cycles received. All had received a minimum of four cycles. A flow diagram of patient selection is demonstrated in Figure 1.

This study was conducted following the declaration of Helsinki regarding ethical principles for medical research. ${ }^{23}$

\section{Pathologic and morphologic study}

All pathology slides of the selected patients' core biopsies and post-surgery breast mass and lymph nodes were reviewed by an expert pathologist. Tumor size, its histologic and nuclear grade (score 1-3), skin and nipple, lymph vascular or dermal lymph vascular involvements, number of involved lymph nodes, and extra nodal extension were evaluated. Tumoral and stromal lymphocytic infiltration (SLI and TLI respectively) were also evaluated on hematoxylin and eosin-stained sections of available core biopsies. TLI and SLI were respectively calculated evaluating percentage of lymphocyte infiltration into tumor cells and into stromal bed without invasion to tumor cells. Threshold used for comparison of low and medium-high TLI and SLI was $10 \%$ according to previously published data. ${ }^{9,24,25}$

\section{Immunohistochemical analysis}

Monoclonal antibodies against estrogen and progesterone receptors (ER, PR), HER2, ki67 and p53 (Biocare Medical, Concord, CA, USA) were used for staining. ER and PR expression were accounted as positive if more than $1 \%$ of the cells were stained. ${ }^{26}$ Detailed interpretation regarding the extent (weak, moderate or strong) and number of cells stained were recorded. HER2 amplification was considered positive if more than $10 \%$ of membrane cells were uniformly stained. Two plus score of HER2 expression was further evaluated for fluorescent in situ hybridization to determine HER2 positivity. $^{27}$ ki67 staining was recorded as the percentage of stained cells out of all cancer cells without considering the intensity of staining; however a threshold of $20 \%$ was used for estimation of high and low ki67 expression. ${ }^{28}$ p53 values were also reported as either positive or negative using the previously reported $10 \%$ cut point. $^{18}$

Afterwards according to definitions for classification of breast cancer molecular subtypes (Table 1), tumors were
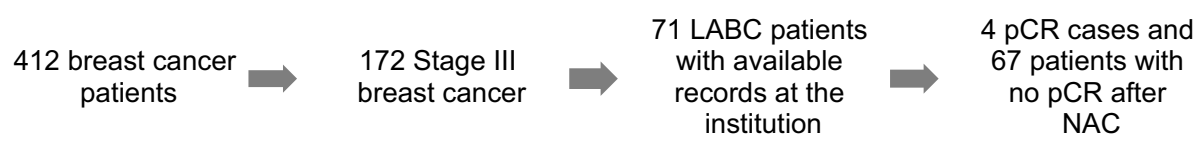

Figure I Flow diagram of patient selection. 
Table I Definition of breast cancer molecular subtypes

\begin{tabular}{|c|c|c|c|c|c|}
\hline Tumor molecular subtype & \multicolumn{3}{|l|}{ Luminal } & HER2 enriched & Triple negative \\
\hline \multirow[t]{3}{*}{ Gene expression pattern } & \multicolumn{3}{|l|}{ ER/PR positive } & \multirow{3}{*}{$\begin{array}{l}\text { ER/PR negative } \\
\text { HER2 positive }\end{array}$} & \multirow{3}{*}{$\begin{array}{l}\text { ER/PR negative } \\
\text { HER2 negative }\end{array}$} \\
\hline & Luminal A & Luminal B & Luminal HER2 & & \\
\hline & $\begin{array}{l}\text { ER/PR positive } \\
\text { HER2 negative }\end{array}$ & $\begin{array}{l}\text { ER positive } \\
\text { PR low positive } \\
\text { HER2 negative }\end{array}$ & $\begin{array}{l}\text { ER positive } \\
\text { PR low positive } \\
\text { HER2 positive }\end{array}$ & & \\
\hline
\end{tabular}

Abbreviations: ER, estrogen receptor; PR, progesterone receptor.

divided as luminal, luminal HER2, HER2 enriched or triple negative. Luminal subtype was further divided to A or $\mathrm{B}$ according to the extent of ER and PR expression.

\section{Evaluation of $p C R$}

According to the 8th edition of the American Joint Committee on Cancer (AJCC) breast cancer staging system, ${ }^{10}$ pCR was evaluated as the absence of any residual invasive cancer following evaluation of the total resected breast tissue and all involved lymph nodes after completion of NAC. In situ carcinoma (DCIS) was allowed in the pCR evaluation. Aside from pCR, residual cancer burden $(\mathrm{RCB})^{29}$ and Miller and Payne grading were also used for evaluation of response to NAC. The latter grading was evaluated according to reduction in tumor cellularity; Grade 1 representing no reduction and grade 5 indicating no residual invasive cancer in the resected tissue. ${ }^{30}$ According to the two latter classifications of response to chemotherapy, patients were categorized. RCB-0 (indicating no residual tumor), RCB-I and Miller and Payne grade 4 and 5, were considered as good response to chemotherapy. On the other hand RCB-II, RCB-III along with Miller and Payne grades 1, 2 and 3 were accounted as poor response.

\section{Statistical analysis}

Statistical analysis was performed using SPSS version 15.0. All data that were categorized as demographic variables and their significance in difference were analyzed using the chisquare and Fisher's exact tests. Logistic regression was used to evaluate the association of different variables with achieving $\mathrm{pCR}$ in different subtypes of breast cancer.

\section{Ethics approval and informed consent}

Institutional review board committee approval was obtained from the Shiraz University of Medical Sciences
Ethics Committee (95-01-36-13927) and written informed consent was collected from patients.

\section{Results}

Detailed data of patients and the characteristics of their tumoral tissue are elaborated in Table 2.

The molecular status of breast cancer in the study population with their percentages is shown in Table 3 . pCR rate was four out of 71 patients $(5.6 \%)$. Multinomial regression was applied in order to find the association of different tumor characteristics and also p53 and ki67 markers in the four subtypes of breast cancer achieving pCR; large confidence intervals were achieved and therefore not valuable for further investigation. The detailed frequency of ki67 positivity, p53 expression, TLI and SLI in different tumor subtypes is elaborated in Table 3.

Our results indicate that ki67, TLI and SLI have significant association with triple negative tumor subtype with a $P$-value of $0.011,0.002$ and 0.014 respectively. Also, both TLI and SLI showed significant association with each other $(P<0.001)$. The positivity of proliferation marker, ki67 showed significant association with positivity in p53 $(P<0.001)$. The percentage of ki67 positivity and its distribution in the study population is shown in Figure 2. As results indicate, pCR was achieved in only four cases. Aside from $\mathrm{pCR}$, the results of response to NAC according to RCB and Miller and Payne grading in different molecular subtypes are elaborated in Table 4. As shown, poor response to NAC is predominant in all four subtypes, showing dominancy in higher RCBs and lower Miller and Payne grading. However $21 \%$ of patients showed partial response to NAC. This partial response is regarded as down staging of tumor from stage III to stage II or I. Association of ki67, p53, TLI and SLI with response (good vs poor) according to $\mathrm{RCB}$ and Miller and Payne grading is shown in Table 5. A significant association was seen between p53 expression with good 
Table 2 Clinical and histologic data of NAC-treated LABC patients

\begin{tabular}{|c|c|c|}
\hline Characteristics & $\begin{array}{l}\text { Number of } \\
\text { patients } \\
(n=7 I)\end{array}$ & $\begin{array}{l}\text { Percentage } \\
\text { of patients }\end{array}$ \\
\hline \multicolumn{3}{|l|}{ Age (years) } \\
\hline $20-29$ & 2 & 2.8 \\
\hline $30-39$ & 12 & 16.9 \\
\hline $40-49$ & 32 & 45.1 \\
\hline $50-59$ & 14 & 19.7 \\
\hline$\geq 60$ & 11 & 15.5 \\
\hline \multicolumn{3}{|l|}{ Largest tumor diameter } \\
\hline$<2 \mathrm{~cm}$ & 24 & 33.8 \\
\hline $2-5 \mathrm{~cm}$ & 32 & 45.1 \\
\hline$>5 \mathrm{~cm}$ & 15 & 21.1 \\
\hline \multicolumn{3}{|l|}{ Tumor grade (Nottingham) } \\
\hline I & 18 & 25.4 \\
\hline II & 35 & 49.3 \\
\hline III & 18 & 25.4 \\
\hline \multicolumn{3}{|l|}{ Nuclear grade } \\
\hline I & 10 & 14.1 \\
\hline II & 25 & 35.2 \\
\hline III & 36 & 50.7 \\
\hline \multicolumn{3}{|l|}{$\begin{array}{l}\text { Number of lymph nodes } \\
\text { involved }\end{array}$} \\
\hline 0 (N0) & 15 & 21.1 \\
\hline $\mathrm{I}-3(\mathrm{NI})$ & 9 & 12.7 \\
\hline 4-9 (N2) & 21 & 29.6 \\
\hline$\geq 10$ (N3) & 26 & 36.6 \\
\hline \multicolumn{3}{|l|}{ Nodal status } \\
\hline Positive & 56 & 78.9 \\
\hline Negative & 15 & 21.1 \\
\hline \multicolumn{3}{|l|}{ Nipple involvement } \\
\hline Positive & 15 & 21.1 \\
\hline Negative & 56 & 78.9 \\
\hline \multicolumn{3}{|l|}{ Skin involvement } \\
\hline Positive & 17 & 23.9 \\
\hline Negative & 54 & 76.1 \\
\hline \multicolumn{3}{|l|}{ Lymphocytic infiltration } \\
\hline TLI & 31 & 43.7 \\
\hline SLI & 30 & 42.3 \\
\hline \multicolumn{3}{|l|}{ Extra nodal extension } \\
\hline Positive & 26 & 36.6 \\
\hline Negative & 45 & 63.4 \\
\hline \multicolumn{3}{|l|}{ TNM staging } \\
\hline 1 & 8 & 11.3 \\
\hline II & 8 & 11.3 \\
\hline III & 55 & 77.5 \\
\hline
\end{tabular}

Abbreviations: NAC, neoadjuvant chemotherapy; LABC, locally advanced breast cancer; TLI, tumoral lymphocytic infiltration; SLI, stromal lymphocytic infiltration. response to NAC according to both RCB and Miller and Payne grading ( $P=0.022, P=0.019$ respectively). Nodal status also had a significant association with nuclear grade in different tumor subtypes $(P=0.036)$. According to the results, $16.4 \%$ of patients had pathologic nodal response.

\section{Discussion}

Considering the heterogeneity of breast cancer, it is important to know its molecular status and related characteristics in approach to its treatment. In this study we decided to evaluate different characteristics and original molecular status of LABC patients that had undergone at least four cycles of NAC.

Data regarding frequency of molecular subtypes of breast cancer are different. Generally luminal A accounts for $30-40 \%$ and Luminal B for $20-30 \%$ of total breast cancers. HER 2 positivity and triple negativity are roughly detected in $12-20 \%$ and $15 \%$ of the breast cancer population respectively. ${ }^{18}$ Our report indicates comparable frequency of luminal subtypes and a higher HER2 positivity compared to global reports; however high rates of HER2 expression was also observed in another study on Iranian Kurdish women. ${ }^{31}$ In a recent study in Eastern Morocco, contrary to global reports and our study results, the majority of breast cancer patients were of the triple negative subtype, followed by HER2 positive, luminal $\mathrm{B}$, and luminal $\mathrm{A},{ }^{32}$ although in a study on ethnicity and subtypes of breast cancer, triple negative subtype is reported to have the highest rate in non-Hispanic black race and hormone receptor positive/HER2 negative subtype being the most prevalent in all ethnic populations as was reported in our study. ${ }^{33}$

As mentioned, $\mathrm{pCR}$ rate was insignificant in our study population. One main reason for this low $\mathrm{pCR}$ rate can be incorporation of breast/axilla in the definition of $\mathrm{pCR}$ rather than only breast. ${ }^{34}$ From the four pCR cases, all had lower primary lymph node involvement and smaller tumor size compared to the non-pCR cases. These could be accounted as possible factors involved in achieving response. Three were HER2 positive. As reported in previous studies, ${ }^{2,13,34,35}$ HER2 positive/ER negative subtypes respond better to NAC and have more appreciable pathologic response. Targeted therapy in case of HER2 positivity can be one reason for better response rates in this group. ${ }^{8,34}$ On the other hand ER positivity and higher histologic grades have been associated with poor response rates. ${ }^{36}$ In our report, the HER2 negative/hormone receptor 
Table 3 Molecular status, ki67 positivity, p53 expression, TLI, SLI of tumor in LABC patients

\begin{tabular}{|c|c|c|c|c|c|}
\hline $\begin{array}{l}\text { Molecular expression } \\
n=7 \mid\end{array}$ & Number of patients n (\%) & $\begin{array}{l}\text { ki67 positivity } \\
\text { n (\%) }\end{array}$ & $\begin{array}{l}\text { p53 expression } \\
\text { n (\%) }\end{array}$ & $\begin{array}{l}\text { TLI } \\
\text { n (\%) }\end{array}$ & $\begin{array}{l}\text { SLI } \\
\text { n (\%) }\end{array}$ \\
\hline \multicolumn{6}{|l|}{ Hormonal status } \\
\hline ER positive & $5 I(7 \mid .8)$ & $13(25.5)$ & $28(54.9)$ & $16(31.4)$ & $15(29.4)$ \\
\hline PR positive & $47(66.2)$ & $12(25.5)$ & $24(51.5)$ & $15(31.9)$ & $14(29.8)$ \\
\hline \multicolumn{6}{|l|}{ HER2 status } \\
\hline positive & $26(36.6)$ & $12(46.2)$ & $19(73.1)$ & $13(50)$ & $12(46.2)$ \\
\hline \multicolumn{6}{|l|}{ Molecular subtype } \\
\hline Luminal & $35(49.3)$ & $8(22.9)$ & $15(42.9)$ & $9(25.7)$ & $10(28.6)$ \\
\hline Luminal HER2 & $16(22.5)$ & $5(3 \mid .3)$ & $13(81.3)$ & $7(43.8)$ & $5(31.3)$ \\
\hline HER2 enriched & $10(14.1)$ & $7(70)$ & $6(60)$ & $6(60)$ & $7(70)$ \\
\hline Triple negative & $10(14.1)$ & $8(80)$ & $7(70)$ & $9(90)$ & $8(80)$ \\
\hline
\end{tabular}

Abbreviations: LABC, locally advanced breast cancer; TLI, tumoral lymphocytic infiltration; SLI, stromal lymphocytic infiltration; ER, estrogen receptor; PR, progesterone receptor.

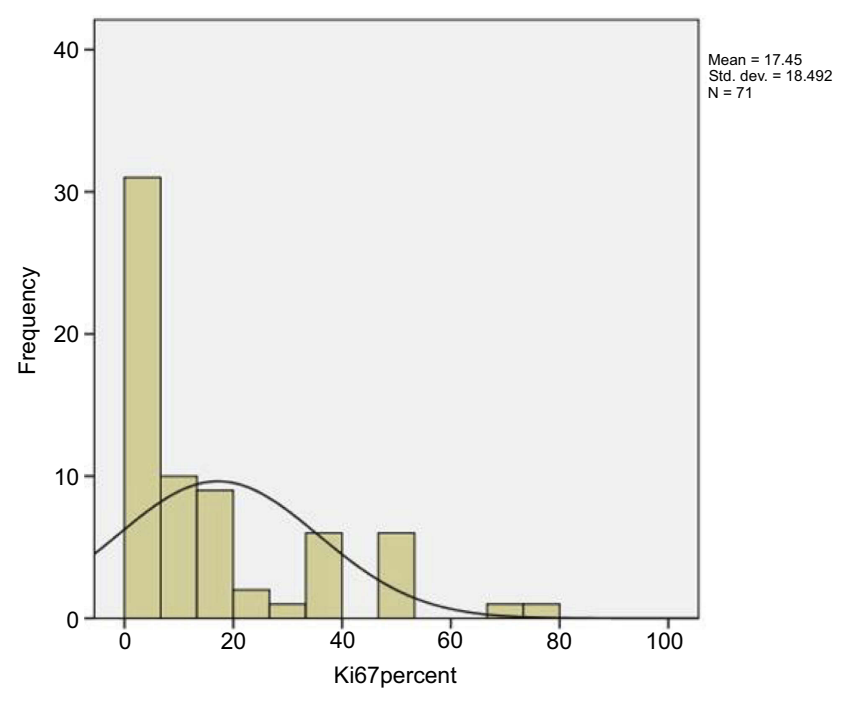

Figure 2 ki67 distribution in $L A B C$ breast cancer patients. Abbreviations: $L A B C$, locally advanced breast cancer.

positive tumor with $\mathrm{pCR}$ had low histologic grade. However heterogeneity in LABC and multiple factors involved in outcome make it difficult to decisively attribute response to a single factor. Aside from $\mathrm{pCR}$, response rates according to RCB and Miller and Payne scoring also approve of higher response rates in HER2-positive tumors. Poor response to NAC was seen predominantly in luminal subtypes. High response rates in the HER2-positive and triple-negative subtypes have been associated to higher proliferative genes. ${ }^{37}$ On the contrary, ER expression has been inversely associated with proliferation of breast tumor. ${ }^{12}$ As presumed, high tumor proliferation rates can lead to better response to chemotherapy. ki67, a proliferation marker, can help predict response to therapy. In our study population high expression of ki67 was observed in the majority of HER2 enriched and triple negative tumor subtypes which is relevant to previous studies. ${ }^{38,39}$ Better response rates in these two subtypes can be due to their higher ki67 expression. Although high ki67 expression was associated with triple negative tumor subtype in our study, it showed no significant association with response to therapy according to RCB and Miller-Payne grading. Its association with tumor subtypes has been variable; in a study by Denkert et al ${ }^{40}$ it has been predictive in all subtypes ${ }^{40}$ with more favorable prognosis in both triple negative and luminal subtypes. ${ }^{39}$ In another study, ki67 showed predictive value in ER positive tumor subtypes being inversely related to ER positivity. ${ }^{12}$ Our results accordingly show low ki67 expression in the majority of luminal A subtypes with high ER expression and poor response to NAC. Different response rates in various studies could also be justified by different unidentified genetic variations in each subtype alone.

In the Miller-Payne grading, the remaining macroscopic or microscopic tumor size is not taken into account and as described, morphologic changes in tumor cells, independent of the chemotherapy regimen chosen, is accounted as response. It is important to emphasize that loss of pathologic tumor cellularity may not always be in accordance with decrease in tumor size indicating that pathologic and clinical response may not be parallel. ${ }^{30}$ However RCB calculation is dependent on residual tumor size as well as tumor cellularity, percentage of DCIS and number of involved lymph nodes with their largest metastasis diameter. ${ }^{29}$ Therefore if exact calculation is done for RCB, it can be accounted as a more specific method of evaluating response. 
Table 4 Response to NAC according to RCB and Miller-Payne scoring in different breast cancer subtypes

\begin{tabular}{|c|c|c|c|c|c|}
\hline $\begin{array}{l}\text { Tumor subtypes } \\
(n=7 I)\end{array}$ & $\begin{array}{l}\text { Luminal } \\
n=35 \text { (\%) }\end{array}$ & $\begin{array}{l}\text { Luminal HER2 } \\
n=16(\%)\end{array}$ & $\begin{array}{l}\text { HER2 enriched } \\
n=10 \text { (\%) }\end{array}$ & $\begin{array}{l}\text { Triple negative } \\
n=10 \text { (\%) }\end{array}$ & Response \\
\hline \multicolumn{6}{|l|}{ RCB } \\
\hline 0 & I (2.9) & $2(12.5)$ & I $(10.0)$ & - & Good \\
\hline I & $10(28.6)$ & $6(37.6)$ & $3(30.0)$ & $2(20.0)$ & Good \\
\hline II & $6(17.1)$ & $3(18.8)$ & $2(20.0)$ & $3(30.0)$ & Poor \\
\hline III & I8 (5 I.4) & $5(3 \mid .3)$ & $4(40.0)$ & $5(50.0)$ & Poor \\
\hline \multicolumn{6}{|l|}{ Miller-Payne } \\
\hline 1 & $19(54.3)$ & $5(3 \mid .3)$ & $4(40.0)$ & $5(50.0)$ & Poor \\
\hline 2 & $3(8.60)$ & $3(18.8)$ & $2(20.0)$ & $3(30.0)$ & Poor \\
\hline 3 & $7(20.0)$ & $2(12.5)$ & I $(10.0)$ & - & Poor \\
\hline 4 & $5(14.3)$ & $4(25.0)$ & $2(20.0)$ & $2(20.0)$ & Good \\
\hline 5 & I (2.9) & $2(12.5)$ & I $(10.0)$ & - & Good \\
\hline
\end{tabular}

Abbreviation: NAC, neoadjuvant chemotherapy; RCB, residual cancer burden.

Table 5 Association of ki67 positivity (cutoff 20\%), p53 expression, TLI and SLI with response to NAC in LABC

\begin{tabular}{|l|l|l|}
\hline $\begin{array}{l}\text { Characteristics } \\
\text { total (n=7 I) }\end{array}$ & $\begin{array}{l}\text { Response } \\
\text { (RCB) } \\
\text { P-value }\end{array}$ & $\begin{array}{l}\text { Response (Miller and } \\
\text { Payne) } \\
\text { P-value }\end{array}$ \\
\hline ki67 positivity & 0.159 & 0.245 \\
P53 expression & 0.022 & 0.019 \\
TLI & 0.646 & 0.646 \\
SLI & 0.432 & 0.746 \\
\hline
\end{tabular}

Note: $P \leq 0.05$ counted as significant.

Abbreviations: SLI, stromal lymphocytic infiltration; TLI, tumoral lymphocytic infiltration; NAC, neoadjuvant chemotherapy.

The prognostic value of $\mathrm{p} 53$ in response to NAC is conflicting, however more recent studies approve its value. $^{15,21,41}$ Methods used for p53 expression can be a limitation to its detection. Some authors prefer gene detection rather than protein as the method of choice. Also small study populations have been another barrier to the discrepancy met in establishing a threshold for p53 expression. $^{21,41} \mathrm{Up}$ to $71 \%$ of HER2-enriched tumors are reported to have p53 mutations, ${ }^{18}$ though this expression was slightly lower in our HER2-enriched tumor population (60\%) (Table 3). This can suggest the existence of other different independent tumorigenesis pathways for breast cancer. In our study, p53 expression showed association with ki67 positivity. Association of ki67 and p53 has been previously observed in triple negative breast cancer patients. $^{42}$ Also overexpression of both ki67 and p53 has been associated with high NAC response rates in the mentioned subtype. ${ }^{15}$ p53 in healthy cells is destined to having a short half-life, therefore it is hardly detected. However in stressed mutated cells, the regulation on its planned destruction is reduced and due to its accumulation and longer half-life its high levels can be detected immunohistochemically. If p53 is dysfunctional due to mutations, natural apoptosis of cancer cells will not take place and mutated DNA remains. As a result uncontrolled cell division leads to high rates of proliferation that can itself be presented as high ki67 expression. ${ }^{43}$ In our study population, p53 expression showed association with good response according to both RCB and Miller-Payne grading. Association of p53 with response to NAC as a prognostic factor has been previously reported ${ }^{15,41}$ although its method of detection, patient characteristics, chemotherapy regimen have been considered as factors that can influence its predictive role.

Nodal status and nuclear grade have also been investigated in association with pathologic and clinical responses in previous studies. ${ }^{2,7-9}$ An association between higher nuclear grades and involvement of lymph nodes in the post NAC breast tissue was observed in our study population as also observed in a similar study. $^{38}$ This finding can help us suggest that higher nuclear grade in the core needle biopsy can predict post NAC nodal metastatic residue.

Host response may also play a role in defeating cancer cells. The amount of lymphocyte gathering in the tumor can be a predictor of response to chemotherapy. In our study population, highest lymphocyte infiltrations were predominantly observed in the triple negative and HER2enriched group that is relevant to previous studies. ${ }^{4-48}$ However Denkert et $\mathrm{al}^{25}$ reported TLI as a predictive marker for response to NAC in all different molecular subtypes, yet it failed to show survival benefit in HER2- 
positive and triple-negative subtypes. ${ }^{25}$ In our study, TLI and SLI showed significant association with triple negative tumor subtype although failed to show association with response due to small number of patients with triple negative subtype and pCR. Strong associations of TLI and SLI in our study and previous studies, ${ }^{2}$ propose the unnecessity of evaluating both in one sample at the same time. Knowing that suppression of the immune systems is an attributing factor to tumor growth, lymphocyte infiltration in core biopsies can be used as an immunity biomarker in predicting response to NAC.

\section{Conclusion}

In this study we were able to show the association of ki67 and $\mathrm{p} 53$ in patients diagnosed with $\mathrm{LABC}$ after receiving NAC. Also ki67 positivity, TLI and SLI were associated to the triple negative subtype and the significant association of TLI with SLI can suggest that they can be representative of one another. From the results of this study we can propose a predictive role for $\mathrm{p} 53$ expression towards better response rates to NAC. Our results indicate that nuclear grade of the primary tumor can be used as a factor in predicting residual tumor in lymph nodes after receiving NAC.

However there exist different limitations to this study. Its retrospective nature limits study population and also the possibility of gathering every baseline data required for patient evaluation throughout his treatment. We should take into account that not every patient's data is complete in the data base and also some patients had to be excluded due to missing pathology biopsies. On the other hand LABC patients without known metastasis are a limited group of breast cancer patients that are not always referred to the affiliated hospital that our data was obtained from.

\section{Data availability}

The data that support the findings of this study are available from the corresponding author upon reasonable request.

\section{Acknowledgments}

This study was financially supported by Shiraz University of Medical Sciences (95-01-36-13927).

\section{Disclosure}

The authors report no conflicts of interest in this work.

\section{References}

1. Vaidya JS, Massarut S, Vaidya HJ, et al. Rethinking neoadjuvant chemotherapy for breast cancer. BMJ. 2018;360:j5913. doi:10.1136/ bmj.j5913

2. Li X, Krishnamurti U, Bhattarai S, et al. Biomarkers predicting pathologic complete response to neoadjuvant chemotherapy in breast cancer. Am J Clin Pathol. 2016;145(6):871-878. doi:10.1093/ajcp/ aqw045

3. Vila J, Mittendorf EA, Farante G, et al. Nomograms for predicting axillary response to neoadjuvant chemotherapy in clinically nodepositive patients with breast cancer. Ann Surg Oncol. 2016;23 (11):3501-3509. doi:10.1245/s10434-016-5277-1

4. Pennisi A, Kieber-Emmons T, Makhoul I, Hutchins L. Relevance of pathological complete response after neoadjuvant therapy for breast cancer. Breast Cancer. 2016;10(BCBCR):S33163. doi:10.4137/ BCBCR.S33163

5. von Minckwitz G, Blohmer JU, Costa SD, et al. Response-guided neoadjuvant chemotherapy for breast cancer. J Clin Oncol. 2013;31 (29):3623-3630. doi:10.1200/JCO.2012.45.0940

6. Von Minckwitz G, Blohmer J, Costa S, et al. Neoadjuvant chemotherapy adapted by interim response improves overall survival of primary breast cancer patients-results of the gepartrio trial. AACR. 2011;(71) (24 Supplement):S3-2.

7. Cortazar P, Zhang L, Untch M, et al. Pathological complete response and long-term clinical benefit in breast cancer: the CTNeoBC pooled analysis. Lancet. 2014;384(9938):164-172. doi:10.1016/S0140-6736 (13)62422-8

8. von Minckwitz G, Untch M, Blohmer J-U, et al. Definition and impact of pathologic complete response on prognosis after neoadjuvant chemotherapy in various intrinsic breast cancer subtypes. $J$ Clin Oncol. 2012;30(15):1796-1804. doi:10.1200/JCO.2011.38.8595

9. Denkert C, Loibl S, Noske A, et al. Tumor-associated lymphocytes as an independent predictor of response to neoadjuvant chemotherapy in breast cancer. J Clin Oncol. 2010;28(1):105-113. doi:10.1200/ JCO.2009.23.7370

10. Giuliano AE, Connolly JL, Edge SB, et al. Breast cancer-major changes in the American Joint Committee on Cancer eighth edition cancer staging manual. CA Cancer J Clin. 2017;67(4):290-303. doi:10.3322/caac. 21393

11. Bhargava R, Beriwal S, Dabbs DJ, et al. Immunohistochemical surrogate markers of breast cancer molecular classes predicts response to neoadjuvant chemotherapy: a single institutional experience with 359 cases. Cancer Interdiscip Int J Am Cancer Soc. 2010;116(6):1431-1439. doi:10.1002/cncr.24876

12. Sueta A, Yamamoto Y, Hayashi M, et al. Clinical significance of pretherapeutic Ki67 as a predictive parameter for response to neoadjuvant chemotherapy in breast cancer; is it equally useful across tumor subtypes? Surgery. 2014;155(5):927-935. doi:10.1016/j. surg.2014.01.009

13. Carey LA, Dees EC, Sawyer L, et al. The triple negative paradox: primary tumor chemosensitivity of breast cancer subtypes. Clin Cancer Res. 2007;13(8):2329-2334. doi:10.1158/1078-0432.CCR06-1109

14. Yoshioka T, Hosoda M, Yamamoto M, et al. Prognostic significance of pathologic complete response and Ki67 expression after neoadjuvant chemotherapy in breast cancer. Breast Cancer. 2015;22(2):185191. doi:10.1007/s12282-013-0474-2

15. Kim T, Han W, Kim MK, et al. Predictive significance of p53, Ki-67, and $\mathrm{Bcl}-2$ expression for pathologic complete response after neoadjuvant chemotherapy for triple-negative breast cancer. $J$ Breast Cancer. 2015;18(1):16-21. doi:10.4048/jbc.2015.18.1.16

16. Kim KI, Lee KH, Kim TR, Chun YS, Lee TH, Park HK. Ki-67 as a predictor of response to neoadjuvant chemotherapy in breast cancer patients. J Breast Cancer. 2014;17(1):40-46. doi:10.4048/jbc.2014. 17.1.40 
17. Dowsett M, Nielsen TO, A'hern R, et al. Assessment of Ki67 in breast cancer: recommendations from the International Ki67 in Breast Cancer working group. J Natl Cancer Inst. 2011;103(22):1656-1664. doi:10.1093/jnci/djr393

18. Tang $\mathrm{P}$, Tse GM. Immunohistochemical surrogates for molecular classification of breast carcinoma: a 2015 update. Arch Pathol Lab Med. 2016;140(8):806-814. doi:10.5858/arpa.2015-0133-RA

19. Lee DS, Kim SH, Kim S, Suh YJ, Kim HK, Shim BY. Prognostic significance of breast cancer subtype and p53 overexpression in patients with locally advanced or high-risk breast cancer treated using upfront modified radical mastectomy with or without postmastectomy radiation therapy. Int J Clin Oncol. 2012;17(5):447455. doi:10.1007/s10147-011-0309-0

20. Lee D-S, Kim SH, Suh YJ, Kim S, Kim HK, Shim BY. Clinical implication of p53 overexpression in breast cancer patients younger than 50 years with a triple-negative subtype who undergo a modified radical mastectomy. Jpn J Clin Oncol. 2011;41(7):854-866. doi:10.1093/jjco/hyr066

21. Bertheau P, Lehmann-Che J, Varna M, et al. p53 in breast cancer subtypes and new insights into response to chemotherapy. Breast. 2013;22:S27-S29. doi:10.1016/j.breast.2013.07.005

22. Carlson RW, Allred DC, Anderson BO, et al. Breast cancer. JNCCN. 2009;7(2):122-192

23. World Medical Association. Declaration of Helsinki: ethical principles for medical research involving human subjects. JAMA. 2013;310 (20):2191. doi:10.1001/jama.2013.281053

24. Lee HJ, Seo J-Y, Ahn J-H, Ahn S-H, Gong G. Tumor-associated lymphocytes predict response to neoadjuvant chemotherapy in breast cancer patients. J Breast Cancer. 2013;16(1):32-39. doi:10.4048/ jbc.2013.16.1.32

25. Denkert C, von Minckwitz G, Darb-Esfahani S, et al. Tumour-infiltrating lymphocytes and prognosis in different subtypes of breast cancer: a pooled analysis of 3771 patients treated with neoadjuvant therapy. Lancet Oncol. 2018;19(1):40-50. doi:10.1016/S1470-2045 (17)30904-X

26. Hammond MEH, Hayes DF, Dowsett M, et al. American Society of Clinical Oncology/College of American Pathologists guideline recommendations for immunohistochemical testing of estrogen and progesterone receptors in breast cancer (unabridged version). Arch Pathol Lab Med. 2010;134(7):e48-e72.

27. Wolff AC, Hammond MEH, Hicks DG, et al. Recommendations for human epidermal growth factor receptor 2 testing in breast cancer: American Society of Clinical Oncology/College of American Pathologists clinical practice guideline update. Arch Pathol Lab Med. 2013;138(2):241-256. doi:10.5858/arpa.2013-0953-SA

28. Goldhirsch A, Winer EP, Coates A, et al. Personalizing the treatment of women with early breast cancer: highlights of the St Gallen International Expert Consensus on the Primary Therapy of Early Breast Cancer 2013. Ann Oncol. 2013;24(9):2206-2223. doi:10.1093/annonc/mdt303

29. Symmans WF, Peintinger F, Hatzis C, et al. Measurement of residual breast cancer burden to predict survival after neoadjuvant chemotherapy. J Clin Oncol. 2007;25(28):4414-4422. doi:10.1200/ JCO.2007.10.6823

30. Ogston KN, Miller ID, Payne S, et al. A new histological grading system to assess response of breast cancers to primary chemotherapy: prognostic significance and survival. Breast. 2003;12(5):320-327.

31. Payandeh M, Sadeghi M, Sadeghi E, Madani S-H. Expression of p53 breast cancer in Kurdish women in the West of Iran: a reverse correlation with lymph node metastasis. Asian Pac J Cancer Prev. 2016;17(3):1261-1264.

32. Errahhali ME, Errahhali ME, Ouarzane M, El Harroudi T, Afqir S, Bellaoui M. First report on molecular breast cancer subtypes and their clinico-pathological characteristics in Eastern Morocco: series of 2260 cases. BMC Women's Health. 2017;17(1):3. doi:10.1186/ s12905-016-0361-z
33. Kohler BA, Sherman RL, Howlader N, et al. Annual report to the nation on the status of cancer, 1975-2011, featuring incidence of breast cancer subtypes by race/ethnicity, poverty, and state. JNCI. 2015;107(6). doi:10.1093/jnci/djv048

34. Houssami N, Macaskill P, von Minckwitz G, Marinovich ML, Mamounas E. Meta-analysis of the association of breast cancer subtype and pathologic complete response to neoadjuvant chemotherapy. Eur J Cancer. 2012;48 (18):3342-3354. doi:10.1016/j.ejca.2012.05.023

35. Rouzier R, Perou CM, Symmans WF, et al. Breast cancer molecular subtypes respond differently to preoperative chemotherapy. Clin Cancer Res. 2005;11(16):5678-5685. doi:10.1158/1078-0432.CCR04-2421

36. Mathieu M-C, Rouzier R, Llombart-Cussac A, et al. The poor responsiveness of infiltrating lobular breast carcinomas to neoadjuvant chemotherapy can be explained by their biological profile. Eur $J$ Cancer. 2004;40(3):342-351.

37. Sørlie T, Perou CM, Tibshirani R, et al. Gene expression patterns of breast carcinomas distinguish tumor subclasses with clinical implications. Proc Natl Acad Sci. 2001;98(19):10869-10874. doi:10.1186/ 1471-2407-11-486

38. Shokouh TZ, Ezatollah A, Barand P. Interrelationships between Ki67, HER2/neu, p53, ER, and PR status and their associations with tumor grade and lymph node involvement in breast carcinoma subtypes: retrospective-observational analytical study. Medicine. 2015;94:32. doi:10.1097/MD.0000000000000874

39. Fasching PA, Heusinger K, Haeberle L, et al. Ki67, chemotherapy response, and prognosis in breast cancer patients receiving neoadjuvant treatment. BMC Cancer. 2011;11(1):486. doi:10.1186/14712407-11-486

40. Denkert C, Loibl S, Müller B, et al. Ki67 levels as predictive and prognostic parameters in pretherapeutic breast cancer core biopsies: a translational investigation in the neoadjuvant GeparTrio trial. Annals of Oncology. 2013;24(11):2786-2793. doi:10.1093/annonc/mdt350

41. von Minckwitz G, Sinn H-P, Raab G, et al. Clinical response after two cycles compared to HER2, Ki-67, p53, and bcl-2 in independently predicting a pathological complete response after preoperative chemotherapy in patients with operable carcinoma of the breast. Breast Cancer Res. 2008;10(2):R30. doi:10.1186/bcr1989

42. Payandeh M, Malayeri R, Sadeghi M, Sadeghi E, Gholami F. Expression of p53 and Ki67 in the patients with triple negative breast cancer and invasive ductal carcinoma. Am J Cancer Prev. 2015;3:58-61.

43. Riley T, Sontag E, Chen P, Levine A. Transcriptional control of human p53-regulated genes. Nat Rev Mol Cell Biol. 2008;9(5):402. doi:10.1038/nrm2395

44. Mao Y, Qu Q, Zhang Y, Liu J, Chen X, Shen K. The value of tumor infiltrating lymphocytes (TILs) for predicting response to neoadjuvant chemotherapy in breast cancer: a systematic review and metaanalysis. PLoS One. 2014;9(12):e115103. doi:10.1371/journal. pone. 0115103

45. Loi S, Michiels S, Salgado R, et al. Abstract S1-05: tumor infiltrating lymphocytes (TILs) indicate trastuzumab benefit in early-stage HER2-positive breast cancer (HER2+ BC). Cancer Res. 2013;73(24 Suppl):S1-05.

46. Ono M, Tsuda H, Shimizu C, et al. Tumor-infiltrating lymphocytes are correlated with response to neoadjuvant chemotherapy in triplenegative breast cancer. Breast Cancer Res Treat. 2012;132(3):793805. doi:10.1007/s10549-011-1554-7

47. West NR, Milne K, Truong PT, Macpherson N, Nelson BH, Watson $\mathrm{PH}$. Tumor-infiltrating lymphocytes predict response to anthracycline-based chemotherapy in estrogen receptor-negative breast cancer. Breast Cancer Res. 2011;13(6):R126. doi:10.1186/bcr3052

48. Hwang HW, Jung H, Hyeon J, et al. A nomogram to predict pathologic complete response (pCR) and the value of tumor-infiltrating lymphocytes (TILs) for prediction of response to neoadjuvant chemotherapy (NAC) in breast cancer patients. Breast Cancer Res Treat. 2019;173(2):255-266. 


\section{Publish your work in this journal}

Cancer Management and Research is an international, peer-reviewed open access journal focusing on cancer research and the optimal use of preventative and integrated treatment interventions to achieve improved outcomes, enhanced survival and quality of life for the cancer patient.
The manuscript management system is completely online and includes a very quick and fair peer-review system, which is all easy to use. Visit http://www.dovepress.com/testimonials.php to read real quotes from published authors.

Submit your manuscript here: https://www.dovepress.com/cancer-management-and-research-journal 